\title{
Kidney injury molecule-1: A potential marker of renal recovery after laparoscopic sleeve gastrectomy
}

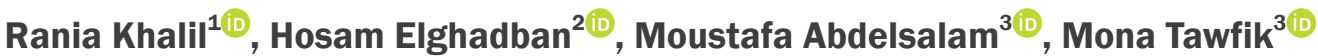 \\ 'Biochemistry Department, Faculty of Pharmacy, Delta University for Science and Technology, Gamasa, Egypt \\ ${ }^{2}$ General Surgery Department, Faculty of Medicine, Mansoura University, Mansoura, Egypt \\ 3Internal Medicine Department, Mansoura Nephrology and Dialysis Unit, Faculty of Medicine, Mansoura University, Mansoura, Egypt
}

Background: Bariatric surgeries were reported to improve diabetes and hypertension; however, the effect on renal recovery has not been fully explored. The aim of this study was to evaluate the effect of laparoscopic sleeve gastrectomy (LSG) in morbidly obese patients on renal function, degree of albuminuria, and kidney injury molecule-1 (KIM-1) level.

Methods: This was a prospective observational study conducted at Mansoura University Hospitals from January to June 2017. Forty-four morbidly obese patients (29 females and 15 males) who met the 1991 WHO criteria for obesity surgery were included. Patients underwent surgical LSG for treatment of morbid obesity, and all were followed for 6 months after surgery. Demographic, clinical, and laboratory data were collected and compared before and after surgery. Primary endpoints were the differences of albuminuria, estimated glomerular filtration rate (eGFR) and serum KIM-1 between baseline (pre-surgery) and 6-month post-surgery values.

Results: Six-month post-surgery data showed significant reduction of body mass index, HbA1c, microalbuminuria, and serum KIM-1, and a significant increase in eGFR (all, $P<0.001$ ). The serum KIM-1 level positively correlated with microalbuminuria and serum creatinine $(r=0.596, P=0.001$ and $r=0.402, P=0.034$, respectively). Postoperative data showed that patients with microalbuminuria had significantly lower eGFR and higher KIM-1 values than those without microalbuminuria ( $P=0.003$ and 0.049 , respectively).

Conclusion: We showed potential benefits of LSG against obesity-associated kidney damage. This is evidenced by improving eGFR and reducing levels of both KIM-1 and microalbuminuria. The serum level of KIM-1 may be a potential marker for renal recovery after LSG.

Keywords: Albuminuria, Kidney injury molecule-1, Laparoscopic sleeve gastrectomy, Obesity

Received October 14, 2019; Revised February 27, 2020; Accepted February 27, 2020

Edited by Soo Wan Kim, Chonnam National University, Gwangju, Republic of Korea

Correspondence: Mona Tawfik

Internal Medicine Department, Mansoura Nephrology and Dialysis Unit, Faculty of Medicine, Mansoura University, Mansoura University Hospitals, 60 El Gomhoureya St. El Mansoura, Dakahlia Governorate 35516, Egypt. E-mail: mona_tawfik20oog9@yahoo. com

Copyright (C) 2020 by The Korean Society of Nephrology

(a) This is an open-access article distributed under the terms of the Creative Commons Attribution Non-Commercial License (http://creativecommons.org/ licenses/by-nc-nd/4.0/), which permits unrestricted non-commercial use, distribution, and reproduction in any medium, provided the original work is properly cited. 


\section{Introduction}

Laparoscopic sleeve gastrectomy (LSG) has been approved by the American Society for Metabolic and Bariatric Surgery (ASMBS) and the American College of Surgeons (ACS) as a treatment for obesity and its related comorbidities. Sleeve gastrectomy involves removal of approximately $80 \%$ of the stomach by transecting the greater curvature of the stomach, which creates a gastric "sleeve" tube in continuity with the esophagus and duodenum. When other conservative measurements failed, bariatric surgery offered substantial and sustainable weight loss and a reduction in obesity-related comorbidities [1].

LSG is a restrictive procedure in which gastric volume is reduced to 60 to $100 \mathrm{~mL}$. This permits intake of only small amounts of food, resulting in a feeling of early satiety during a meal [2]. The effect of LSG on endogenous ghrelin level may also explain LSG success. Ghrelin, a hunger-regulating peptide hormone, is produced by $\mathrm{P} /$ D1 cells found mainly in the fundus of the stomach. By resecting the fundus in LSG, the majority of ghrelin-producing cells is removed. This will reduce plasma ghrelin level and subsequently, will alleviate hunger sensation, that aid to weight loss [3].

LSG has various advantages that include its technical simplicity, shorter operative time, preservation of the pylorus, and maintenance of normal continuity of bowel [4]. Long-term weight loss after LSG is equal to that of laparoscopic Roux-en-Y gastric bypass (LRYGB), which has been suggested to be the gold standard procedure for morbid obesity $[5,6]$.

As recently determined, LSG not only treats obesity, but also is associated with improvement in type 2 diabetes mellitus (DM) and hypertension (HTN). Both of these conditions are independent risk factors for development of chronic kidney disease (CKD), especially in patients with poorly controlled diabetic state. The major indices of kidney damage and dysfunction are serum creatinine, glomerular filtration rate (GFR), and urinary albumin/ creatinine ratio, which are affected by glucose metabolism and insulin resistance even prior to development of diabetes [7]. These markers have some limitations when used as early biomarkers for kidney damage, as they reflect an already existing underlying kidney disease. To better identify individuals with increased risk for kidney disease, there is a need for biomarkers that represent early signs of kidney damage [8]. Several biomarkers such as neutrophil gelatinase-associated lipocalin, kidney injury molecule-1 (KIM-1), and liver-type fatty acid-binding protein were recently evaluated in their abilities to detect renal insults earlier than is currently possible with traditional biomarkers [9-12].

KIM-1 is a membrane protein expressed at minute level in the healthy kidney. After kidney injury, the extracellular portion of the KIM-1 protein is cleaved, rapidly enters the tubular lumen, and then is detectable in the urine. KIM-1 is considered by some researchers a sensitive biomarker of tubular damage and is strongly associated with tubulointerstitial inflammation and fibrosis. Compared to any of the conventional diagnostic markers, KIM- 1 is not only an early biomarker of acute kidney injury, but also has a potential role in predicting long-term renal outcomes $[13,14]$.

Obesity is one of the risk factors of development of CKD on its own or in conjunction with its association with comorbid conditions like DM and HTN. Obesity-related renal disease is usually asymptomatic and difficult to diagnose early [14]. In the current study, we hypothesized that LSG-aided weight reduction could improve renal function; this improvement could be detected using KIM1 and other traditional nephropathy markers. The aim of this study was to explore the effects of LSG on renal function, KIM-1 level, degree of microalbuminuria, and other nephropathy markers. This aim included assessment of LSG's reno-protective effect against the pathogenic mechanism associated with morbid obesity.

\section{Methods}

\section{Study design and inclusion criteria}

This was a prospective observational study that included 44 morbidly obese patients who met the 1991 World Health Organization (WHO) criteria for obesity surgery. These criteria included age between 18 and 65 years old with body mass index (BMI) above $40 \mathrm{~kg} / \mathrm{m}^{2}$ without comorbidities or $35 \mathrm{~kg} / \mathrm{m}^{2}$ with one of the obesity-related comorbidities. Failure of other weight loss methods was also required. The included patients were enrolled in the study and underwent surgical LSG for treatment of obesity. 


\section{Exclusion criteria}

Patients with severe diabetic complications or associated medical conditions such as blindness, end-stage renal failure, liver cirrhosis, malignancy, chronic congestive heart failure, unstable angina pectoris, pregnancy, and psychological conditions that hamper patient cooperation were excluded. Those experiencing specific contraindications to obesity surgery such as chronic diarrhea, alcoholism, inflammatory bowel disease, uncontrolled coagulopathy, nephrotic syndrome, drug addiction, and unwillingness to undergo long-term follow-up were also excluded from the study.

\section{Patient and public involvement}

Both patients and public representatives were involved in recruitment methods. Patients who agreed to be enrolled in the study were followed up for 6 months after LSG. All patients were informed of the study results during their follow-up visits; feedback on the summary of these results was also provided to the patients. All subjects were informed of the study's aims and methods, and informed consent was obtained. All patients were recruited from Mansoura University Hospitals, Safa Hospital, Damietta, and Elkhire Hospital, Mansoura, Egypt, under supervision of the second author between January 2017 and June 2017. The study was approved by the research ethics committee at the Faculty of Pharmacy, Delta University for Science and Technology, Gamasa, Egypt, with registration number FPDU18/2018. The protocol complied with the Ministry of Health, Egypt, and was carried out in accordance with Mansoura University Hospitals guidelines. Informed consent was obtained from each study participant.

\section{Clinical and laboratory assessment}

All patients underwent complete clinical assessment. The weight and height of patients were measured, and BMI was calculated by dividing the patient weight in kilograms $(\mathrm{kg})$ by patient height in meters squared $\left(\mathrm{m}^{2}\right)$. Detailed patient histories were recorded.

Control blood and urine samples were collected before surgery. Blood and urine were re-sampled at the end of the 6 -month postoperative period. Blood samples were drawn by venous arm punctures from patients after an overnight fast. Five $\mathrm{mL}$ of blood was added immediately to a plain tube, and the serum was separated by centrifugation at 4,000 rpm for 15 minutes using a 5,804 centrifuge (Eppedorf Company, Germany). Blood samples were used to measure hemoglobin Alc (HbAlc) (\%), urea nitrogen, uric acid, creatinine, and KIM-1. 24-hour urine samples were collected for urinary albumin quantification.

Glycemic control was assessed by measuring HbAlc\% by the Getine110 system (Biotech Inc., Hamburg, Germany) according to the method described by [15] the manufacturer of commercial kits obtained from Biotech Inc. Serum urea nitrogen [16], uric acid [17], and creatinine [18], were determined by Hitachi Cobas c systems using commercial kits obtained from Roche Diagnostics $\mathrm{GmbH}$, Mannheim, Germany. All measurements followed kit manufacturer instructions.

Serum KIM-1 was assessed by enzyme-linked immunosorbent assay (ELISA) technique using a commercial kit obtained from NOVA (Bioneovan Co. Ltd., Beijing, China). Briefly, the Micro ELISA stripe plates were precoated with an antibody specific to KIM-1, and $50 \mu \mathrm{L}$ of horseradish peroxidase-conjugated antibody specific for KIM-1 was added to each well except the blank control well. The plate was sealed and incubated for 30 minutes at room temperature. Free components were washed away. After washing, $100 \mu \mathrm{L}$ of substrate solution was added to all wells, which were then protected from light, shaken gently, and incubated at room temperature for 15 minutes. The reaction was stopped by adding $50 \mu \mathrm{L}$ of stop solution to all wells. A color change in the well from blue to yellow occurred. The absorbance was measured using a micro-titer plate reader at $450 \mathrm{~nm}$. Serum KIM1 concentration was calculated based on the standard curve and expressed in absolute terms $(\mathrm{pg} / \mathrm{mL})$.

\section{Assessment of renal function}

Estimated GFR (eGFR) was calculated using the CKDEPI (epidemiology equation) formula for those patients with $\mathrm{BMI}<30 \mathrm{~kg} / \mathrm{m}^{2}$ after surgery. The CKD-EPI equation is a single equation in which values are expressed in $\mathrm{ml} /$ $\mathrm{min} / 1.73 \mathrm{~m}^{2}$ [19]. $\operatorname{eGFR}\left(\mathrm{mL} / \mathrm{min} / 1.73 \mathrm{~m}^{2}\right)=141 \times(\mathrm{SCr} / 0.9)^{-1.209} \times 0.993^{\text {age }} \times$ 1.018 (if female)

We used the Salazar Corcoran equation for patients 
who had BMI $\geq 30 \mathrm{~kg} / \mathrm{m}^{2}$, whether before or after surgery, because the CKD-EPI formula fails to properly estimate true renal function. The Salazar Corcoran equation values are expressed in $\mathrm{mL} / \mathrm{min} / 1.73 \mathrm{~m}^{2}$. The equations for men and women are [20].

For men:

[137 - age $] \times\left[(0.285 \times\right.$ weight $(\mathrm{kg}))+\left(12.1 \times\right.$ height $\left.\left(\mathrm{m}^{2}\right)\right]$ divided by $(51 \times \mathrm{SCr})$

For women:

$[146-$ age $] \times\left[(0.287 \times\right.$ weight $(\mathrm{kg}))+\left(9.74 \times\right.$ height $\left.\left(\mathrm{m}^{2}\right)\right]$ divided by $(60 \times \mathrm{SCr})$

\section{Surgical procedure}

After admission, routine antithrombotic prophylaxis was initiated, usually with 4,000 to 6,000 units of lowmolecular-weight heparin according to risk. No bowel preparation was necessary. The patient followed a liquid diet one day before the operation. A single dose of second-generation cephalosporin was administered half an hour before skin incision as ultra-short-term antibiotic prophylaxis.

The sleeve gastrectomy technique began after trocar placement (generally 5-6), retraction of the liver, and identification of the pylorus. A distance of 2 to $4 \mathrm{~cm}$ on the antrum was marked to begin dissection of the greater curvature vessels and ultimate division of the stomach. A calibrating tube $38 \mathrm{~F}$ was used to help standardize the size of the gastric conduit and to prevent over-narrowing of the sleeve. Sequential firings of the linear stapler were continued toward the gastroesophageal junction (angle of His). Then the staple line was reinforced by an oversewing stitch using polydiaxone $2 / 0$. Patients were discharged from the hospital after stabilization of their general condition; the median ( $\min -\max )$ duration of hospital stay was $3(2-5)$ days.

\section{Statistical analysis}

Data were collected, revised, verified, and then edited on a personal computer. The statistical analysis was performed using IBM SPSS Statistics for Windows ver. 21.0 (IBM Corp., Armonk, NY, USA). The normality of data was first tested with the Shapiro-Wilk test. Qualitative data are described using number and percent. Association between categorical variables was tested using
Fisher's exact test or chi-square test when appropriate. Continuous variables are presented as mean \pm standard deviation for parametric data and median (range) for nonparametric data. Unpaired data are compared using Student's $t$ test (for parametric data) and Mann-Whitney test (for nonparametric data), while paired samples were compared using paired sample $t$ test (for parametric data) and Wilcoxon signed-rank test (for nonparametric data).

Significance of the obtained results was considered at a $P$ value of $\leq 0.05$.

Pearson's test was performed to detect correlation with each parameter [21].

\section{Results}

\section{Description of included sample}

The current study comprised 44 obese patients who underwent LSG. The baseline demographic characteristics of the study participants are illustrated in Table 1. The majority of patients enrolled in the study was females $(n=29,65.9 \%)$. The mean age of patients was $32.5 \pm 8.9$ years. The patients enrolled in the study had a BMI of $50.6 \pm 6.7 \mathrm{~kg} / \mathrm{m}^{2}$.

Ten participants $(22.7 \%)$ were diabetic and under treatment with insulin and/or oral hypoglycemic drugs, e.g., metformin $1,000 \mathrm{mg} / \mathrm{d}$, glibenclamide $2.5 \mathrm{mg} / \mathrm{d}$, or glimepiride $3 \mathrm{mg} / \mathrm{d}$. The diagnosis of diabetes was confirmed by history and HbAlc $\geq 6.5 \%$ according to the American Diabetes Association (ADA, 2017). In addition,

Table 1. Baseline demographic characteristics of morbidly obese patients enrolled in the study $(n=44)$

\begin{tabular}{lc}
\hline \multicolumn{1}{c}{ Characteristic } & Value \\
\hline Sex & \\
Male & $15(34.1)$ \\
Female & $29(65.9)$ \\
Age $(\mathrm{yr})$ & $32.5 \pm 8.9$ \\
Body mass index $\left(\mathrm{kg} / \mathrm{m}^{2}\right)$ & $50.6 \pm 6.7$ \\
Total number of diabetic patients & $10(22.7)$ \\
Total number of HTN patients & $12(27.3)$ \\
Duration of treatment in T2DM patients $(\mathrm{yr})^{\mathrm{a}}$ & $6.1 \pm 3.7$
\end{tabular}

Data are presented as number (\%) or mean \pm standard deviation.

HTN, hypertension; T2DM, type 2 diabetes mellitus.

${ }^{\mathrm{a}}$ Total number of diabetic patients $=10$. 
12 patients $(27.3 \%)$ were hypertensive. The patients were followed up closely to confirm adherence to anti-diabetic treatment before and after surgery.

\section{Comparison between pre-surgery and post-surgery data}

Six-month post-surgery, subjects showed a significant reduction in BMI compared to the pre-surgery data. The BMI before the surgery was $50.6 \pm 6.7 \mathrm{~kg} / \mathrm{m}^{2}$ but decreased to $36.2 \pm 5.3 \mathrm{~kg} / \mathrm{m}^{2}$ after surgery. After surgery, seven of $44 \mathrm{pa}-$ tients (15.9\%) had BMI of $30 \mathrm{~kg} / \mathrm{m}^{2}$ or less. A significant postsurgical reduction in $\mathrm{HbAlc} \%$, from $8.4 \pm 1.4 \%$ to $5.2 \pm 0.8 \%$, occurred in diabetic patients. Pre-surgery microalbuminuria, serum urea nitrogen, creatinine, and uric acid were significantly decreased in 6 -month post-surgery samples $(P<0.001)$; and eGFR was significantly increased from $103.0 \pm 14.8 \mathrm{~mL} /$ $\mathrm{min} / 1.73 \mathrm{~m}^{2}$ to $108.4 \pm 14.4 \mathrm{~mL} / \mathrm{min} / 1.73 \mathrm{~m}^{2}$. KIM-1 level was significantly reduced at 6 -month post-surgery. The median pre-surgery KIM-1 value was 1,729.0 (377.5-6,298.4) $\mathrm{pg} / \mathrm{mL}$, while the 6 -month post-surgery value was 477.6 (155.1-1,520.3) pg/mL (Table 2).

The correlations between pre-surgery and post-surgery KIM-1 values, BMI values, and other biochemical parameters are shown in Table 3. KIM-1 exhibited no significant association with BMI, $\mathrm{HbAlc \%}$, urea, uric acid, or eGFR; however, KIM-1 did demonstrate a significant positive association with microalbuminuria and serum creatinine $(\mathrm{r}=$ 0.596 and 0.402 , respectively). The post-surgery KIM- 1 level had a significant positive association with microalbuminuria and creatinine ( $\mathrm{r}=0.391$ and 0.473 , respectively) and a significant negative association with eGFR $(\mathrm{r}=-0.671)$.

\section{Comparison between patients with and without microalbuminuria}

Our patients were subdivided into two groups according to presence or absence of microalbuminuria at initial presentation before LSG. The non-microalbuminuria group was composed of 27 patients; the remaining 17 patients experienced microalbuminuria. The microalbuminuria resolved in 15 of the 17 microalbuminuric patients after LSG. Pre-surgical comparison of microalbuminuria and non-microalbuminuria groups showed no significant differences in BMI, serum creatinine, serum uric acid, eGFR, or KIM-1. DM and HTN prevalence and

Table 3. Correlation of pre-surgery and post-surgery serum KIM-1 with different patient parameters

\begin{tabular}{|c|c|c|c|c|}
\hline \multirow{2}{*}{ Variable } & \multicolumn{2}{|c|}{ Pre-operative (KIM-1) } & \multicolumn{2}{|c|}{ Post-operative (KIM-1) } \\
\hline & $r$ & $P$ value $^{\mathrm{a}}$ & $r$ & $P$ value ${ }^{a}$ \\
\hline Body mass index & 0.099 & 0.624 & 0.296 & 0.126 \\
\hline HbA1C (\%) & 0.191 & 0.330 & 0.141 & 0.465 \\
\hline Microalbuminuria (mg/24 hr) & 0.596 & 0.001 & 0.391 & 0.040 \\
\hline Serum urea nitrogen $(\mathrm{mg} / \mathrm{dL})$ & 0.263 & 0.176 & 0.280 & 0.149 \\
\hline Serum uric acid (mg/dL) & -0.095 & 0.632 & 0.288 & 0.137 \\
\hline eGFR $\left(\mathrm{mL} / \mathrm{min} / 1.73 \mathrm{~m}^{2}\right)$ & -0.07 & 0.722 & -0.671 & $<0.001$ \\
\hline Serum creatinine (mg/dL) & 0.402 & 0.034 & 0.473 & 0.011 \\
\hline
\end{tabular}

eGFR, estimated glomerular filtration rate; HbA1c, hemoglobin Alc; KIM-1, kidney injury molecule-1.

${ }^{a}$ Pearson correlation.

Table 2. Comparison of laboratory data between pre-surgery and 6-month post-surgery in the studied group ( $\mathrm{n}=44)$

\begin{tabular}{lccc}
\hline \multicolumn{1}{c}{ Parameter } & Pre-surgery & Post-surgery & $P$ value \\
\hline Total number of DM patients & $10(22.7)$ & $2(4.5)$ & $0.038^{\mathrm{c}}$ \\
Total number of HTN patients & $12(27.3)$ & $9(20.5)$ & $0.663^{\mathrm{c}}$ \\
Total number microalbuminuric patients & $17(38.6)$ & $2(4.5)$ & $<0.001^{\mathrm{c}}$ \\
Body mass index $\left(\mathrm{kg} / \mathrm{m}^{2}\right)$ & $50.6 \pm 6.7$ & $36.2 \pm 5.3$ & $<0.001^{\mathrm{b}}$ \\
HbA1C $(\%)$ & $8.4 \pm 1.4$ & $5.2 \pm 0.8{\text { (in diabetic patients, } \mathrm{n}=10)^{\mathrm{a}}}^{<0.001^{\mathrm{b}}}$ \\
Microalbuminuria $(\mathrm{mg} / 24 \mathrm{hr})$ & $23 \pm 19$ & $14 \pm 10$ & $<0.001^{\mathrm{b}}$ \\
Serum urea nitrogen $(\mathrm{mg} / \mathrm{dL})$ & $18(11-80)$ & $0.87 \pm 0.1$ & $<0.001^{\mathrm{a}}$ \\
Serum creatinine $(\mathrm{mg} / \mathrm{dL})$ & $0.97 \pm 0.16$ & $5.3 \pm 0.7$ & $<0.001^{\mathrm{b}}$ \\
Serum uric acid $(\mathrm{mg} / \mathrm{dL})$ & $6.2 \pm 1$ & $108.4 \pm 14.4$ & $<0.001^{\mathrm{b}}$ \\
eGFR (mL/min/1.73 m $\left.{ }^{2}\right)$ & $103.0 \pm 14.8$ & $478(155-1,520)$ & $<0.001^{\mathrm{b}}$ \\
Serum KIM-1 $(\mathrm{pg} / \mathrm{mL})$ & $1,729(378-6,298)$ & & $<0.001^{\mathrm{a}}$ \\
\hline
\end{tabular}

Data are expressed as number (\%), mean \pm standard deviation, or median (interquartile range).

DM, diabetes mellitus; eGFR, estimated glomerular filtration rate; HbA1c, hemoglobin Alc; HTN, hypertension; KIM-1, kidney injury molecule-1.

${ }^{a}$ Wilcoxon signed-rank test. ${ }^{b}$ Paired sample $t$ test. ${ }^{c}$ Chi-square test. 
Table 4. Pre-operative comparison of clinical and laboratory data between non-microalbuminuria and microalbuminuria group

\begin{tabular}{lccr}
\hline \multicolumn{1}{c}{ Parameter } & Non-microalbuminuria $(\mathrm{n}=27)$ & Microalbuminuria $(\mathrm{n}=17)$ & \multicolumn{1}{c}{$<$ value } \\
\hline Diabetes mellitus & $1(3.7)$ & $9(52.9)$ & $0.001^{\mathrm{b}}$ \\
Hypertension & $3(11.1)$ & $9(52.9)$ & $0.003^{\mathrm{b}}$ \\
Body mass index $\left(\mathrm{kg} / \mathrm{m}^{2}\right)$ & $49.4 \pm 6.6$ & $52.5 \pm 7.7$ & $0.165^{\mathrm{c}}$ \\
Microalbuminuria $(\mathrm{mg} / 24 \mathrm{hr})$ & $16.1 \pm 3.4$ & $79.4 \pm 38.6$ & $<0.001^{\mathrm{c}}$ \\
Serum urea nitrogen $(\mathrm{mg} / \mathrm{dL})$ & $17(11-25)$ & $20(12-80)$ & $0.012^{\mathrm{a}}$ \\
Serum creatinine $(\mathrm{mg} / \mathrm{dL})$ & $0.96 \pm 0.10$ & $0.99 \pm 0.20$ & $0.519^{c}$ \\
Serum uric acid $(\mathrm{mg} / \mathrm{dL})$ & $6.0 \pm 1.1$ & $6.4 \pm 0.9$ & $0.292^{c}$ \\
eGFR $\left(\mathrm{mL} / \mathrm{min} / 1.73 \mathrm{~m}^{2}\right)$ & $101.6 \pm 11.7$ & $95.4 \pm 18.4$ & $0.179^{c}$ \\
Serum KIM-1 $(\mathrm{pg} / \mathrm{mL})$ & $1,834(378-3,919)$ & $1,407(407-6,298)$ & $0.983^{\mathrm{a}}$ \\
\hline
\end{tabular}

Data are expressed as number (\%), mean \pm standard deviation, or median (interquartile range).

eGFR, estimated glomerular filtration rate; KIM-1, kidney injury molecule-1.

${ }^{a}$ Mann-Whitney $U$ test. ${ }^{b}$ Fisher's exact test. Independent sample $t$ test.

Table 5. Postoperative comparison of clinical and laboratory data between non-microalbuminuria and microalbuminuria group

\begin{tabular}{lccr}
\hline \multicolumn{1}{c}{ Parameter } & Non-microalbuminuria $(\mathrm{n}=27)$ & Microalbuminuria $(\mathrm{n}=17)$ & $P$ value \\
\hline Body mass index $\left(\mathrm{kg} / \mathrm{m}^{2}\right)$ & $34.6 \pm 5.1$ & $38.8 \pm 4.7$ & $0.009^{\mathrm{b}}$ \\
Microalbuminuria $(\mathrm{mg} / 24 \mathrm{hr})$ & $9.7 \pm 4.7$ & $20.9 \pm 11.1$ & $<0.001^{\mathrm{b}}$ \\
Serum urea nitrogen $(\mathrm{mg} / \mathrm{dL})$ & $16(10-21)$ & $18(12-78)$ & $0.085^{\mathrm{a}}$ \\
Serum creatinine $(\mathrm{mg} / \mathrm{dL})$ & $0.86 \pm 0.10$ & $0.89 \pm 0.20$ & $0.288^{\mathrm{b}}$ \\
Serum uric acid $(\mathrm{mg} / \mathrm{dL})$ & $5.4 \pm 0.7$ & $5.5 \pm 0.8$ & $0.747^{\mathrm{b}}$ \\
eGFR $\left(\mathrm{mL} / \mathrm{min} / 1.73 \mathrm{~m}^{2}\right)$ & $113.4 \pm 9.7$ & $100.5 \pm 17.2$ & $0.003^{\mathrm{b}}$ \\
Serum KIM-1 $(\mathrm{pg} / \mathrm{mL})$ & $388(155-996)$ & $755(255-1520)$ & $0.049^{\mathrm{a}}$ \\
\hline
\end{tabular}

Data are expressed as mean \pm standard deviation or median (interquartile range).

eGFR, estimated glomerular filtration rate; KIM-1, kidney injury molecule-1.

${ }^{\mathrm{a}}$ Mann-Whitney $U$ test. ${ }^{\mathrm{b}}$ Independent sample $t$ test.

serum urea level were significantly higher in the microalbuminuria group, as shown in Table 4 . The post-surgical comparison showed significantly lower eGFR and higher BMI and KIM- 1 values in the microalbuminuria group, as in Table 5 The reduction of KIM- 1 after surgery in the microalbuminuria group was $46.3 \%(P=0.013)$, while that in the non-microalbuminuria group was $78.8 \%(P<0.001)$ (Fig. 1).

\section{Discussion}

LSG has emerged as the most effective and most frequently used long-term treatment of morbid obesity. In 2015, LSG accounted for $53.8 \%$ of all bariatric surgeries according to reports from the ASMBS, July 2016 [22]. LSG has proven efficacy in producing dramatic and durable weight loss in obese patients as well as impressive improvements in diabetic complications, dyslipidemia, and HTN [23,24]. Previous reports have demonstrated LSG's

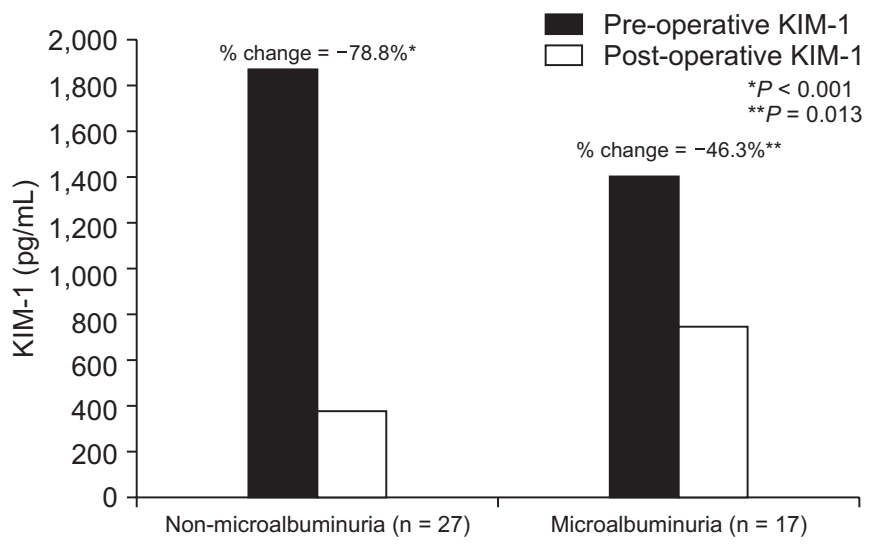

Figure 1. Clustered column chart represents percentage changes of the serum kidney injury molecule-1 (KIM-1) after laparoscopic sleeve gastrectomy in non-microalbuminuria and microalbuminuric group. Probability of Wilcoxon signed-rank test. Columns represent the median values.

beneficial effect on renal function; however, the pathogenic mechanisms underlying this improvement are not 
well understood. In our study, we evaluated both metabolic and nephropathic markers, including KIM-1, in morbidly obese patients before and 6 months after LSG.

In the current study, we found an average $30 \% \mathrm{BMI}$ reduction at 6 months after surgery, and this result is in concordance with Sachdev and colleagues [25]. A previous explanation for the reduction in BMI after LSG was that the reduction was achieved by a combination of mechanical restriction and ghrelin hormonal modulation [2]. Ghrelin is an appetite-modulating peptide that is mainly produced by the cells of the gastric fundus, which is removed by LSG [26]. Of note, our diabetic patients showed less weight loss at 6 -month post-surgery than non-diabetics, a finding that is in concordance with previous reports $[27,28]$.

Similarly, LSG had a noticeable effect on reducing $\mathrm{HbAlc}$ and induced remission of diabetes in the current patients, a finding that was explored by Kalinowski et al [26]. Some authors have observed this improvement in the early postoperative period even without achievement of weight loss, a mechanism that could be explained by hormonal mechanisms (e.g., increased glucagon-like peptide 1 and decreased ghrelin secretion) [3,29]. Unfortunately, data on state of diabetic control in the early postoperative period ( $0-3$ months) were not included in our analysis. This would have served to evaluate the previous observation.

In the current study, microalbuminuria was significantly higher in obese patients with DM or HTN; however, the BMI values did not differ significantly between microalbuminuric and non-microalbuminuric groups. The presence of microalbuminuria in our patients may be explained by the presence of obesity-induced or obesityassociated comorbid conditions such as DM and HTN rather than obesity itself.

Post-surgery data of the current analysis showed a dramatic improvement in serum creatinine and hence eGFR. Holcomb et al [30] previously reported a significant improvement of renal function quantified by eGFR after bariatric surgery (both LSG and LRYGB). We also appreciated the reduction of microalbuminuria 6 months after LSG; improvement of proteinuria and microalbuminuria after weight loss was also explored by previous reports $[31,32]$. Microalbuminuria in the sitting of obesity was thought to be caused by the effects of obesity on inducing systemic inflammation, impaired glucose metabolism and insulin resistance, and high blood pressure [33].

Consistent with our results, Park et al [34] observed a reduction in serum uric acid after bariatric surgery. Park et al [34] explained this observation by the possible interrelated mechanisms between albuminuria and uric acid, theorizing that albuminuria could enhance reabsorption of urate from proximal tubules [35]. This mechanism could explain the reduction of serum uric acid and improvement of albuminuria after weight reduction.

One of the promising biomarkers in detection of early acute kidney injury as well as prediction of CKD is KIM1 ; however, data assessing its value in obese patients are scarce. We reported in the current analysis that KIM-1 values are higher among obese patients and were decreased significantly after LSG. Goknar et al [36] evaluated the panel of early kidney injury biomarkers, including KIM-1, in pediatric obese non-diabetic patients and found significantly higher values of KIM-1 in the obese patients.

The values of KIM- 1 were significantly correlated with microalbuminuria in both pre- and post-surgery data, a finding that could indicate a pattern of tubular injury in our patients. Albumin excretion is primarily related to glomerular injury, but some authors have elucidated a possible role of tubular injury in early occurrence of albuminuria in diabetic obese patients [37]. Our analysis was unable to delineate whether the correlation between KIM-1 and microalbuminuria was a true causal relationship. However, we reported higher KIM-1 values among microalbuminuric and non-microalbuminuric patients. We suggest that the higher level of KIM-1 in non-microalbuminuric patients might be related to subclinical obesity-induced renal injury or its associated comorbid conditions. Previously, Xiao et al [38] found higher KIM-1 level in obese adolescents, even without presence of microalbuminuria. These findings may clarify the possible clinical application of KIM- 1 as a complementary marker for early detection of subclinical obesity-related renal disorder.

Our comprehensive analysis of both microalbuminuric and non-microalbuminuric patients (a group that comprised the majority of diabetic and hypertensive patients) revealed improved weight loss, improved GFR, and reduced KIM-1 in the non-microalbuminuric group. The rate of KIM- 1 reduction was $-78.8 \%$ in the non-microalbuminuric versus $-46.3 \%$ in the microalbuminuric 
group. The preceding findings may indicate more severe injury among patients with microalbuminuria from which the patients had not adequately recovered during the study follow-up period, an observation that indicates the importance of extended follow-up programs for patients to assess the pattern of renal recovery.

We observed in the current study that GFR was not extremely high according to different formulas. These equations did not adjust or correct for risk of obesity overestimation of true renal function. We admit that assessment of renal function is challenging, especially in the setting of obesity and situations with rapid weight loss, and hypothesize that the state of hyperfiltration that is described in obese patients may be linked to a miscalculation of the true renal function. Available observational studies of bariatric surgery that use creatininebased GFR equations for assessing kidney function have important limitations [39].

The renal function of our morbidly obese patients statistically improved after applying LSG, but this significant difference could be clinically insignificant as the absolute difference was small. However, improved kidney function after bariatric surgeries has been described before. In a large meta-analysis, the authors discovered a beneficial effect of bariatric surgeries on preservation of renal function, especially in those with impaired kidney function [40].

\section{Strength and limitations of the study}

This study provided a comprehensive analysis of different nephropathy markers in morbidly obese patients before and 6 months after LSG. These markers include serum creatinine and eGFR, microalbuminuria, serum uric acid, and KIM-1. We acknowledge some limitations. First, there was a limitation associated with GFR estimation. The use of serum creatinine-based equations like CKD-EPI is problematic in the setting of bariatric surgery. These equations could overestimate GFR in obese patients and could cause misinterpretation of GFR for those with rapid weight loss. We attempted to overcome this problem by using two eGFR equations according to the BMI values of our patients. The second limitation is the short follow-up period of 6 months postoperatively; extended evaluation is recommended for such patients to investigate the full pattern of renal recovery. The third limitation is the small sample size of included patients and the heterogeneity of clinical criteria for both obese and obese diabetic patients. Analysis of a larger population with different specific subgroup analysis would have greater clinical significance.

In conclusion, the current study explored the benefits of LSG not only as an anti-obesity, anti-diabetic procedure, but also as a reno-protective one. This was demonstrated by improved GFR, reduced microalbuminuria, and reduced KIM-1. Extended follow-up is recommended after LSG to fully investigate the pattern of renal recovery.

\section{Conflicts of interest}

All authors have no conflicts of interest to declare.

\section{Acknowledgments}

We would like to thank our nursing staff and social workers who helped us in patient selection, education, and follow up.

\section{Authors' contributions}

Hosam Elghadban participated in the data collection and wrote the manuscript. Moustafa Abdelsalam participated in the study design and performed the statistical analysis. Mona Tawfik participated in the conception, analysis, and interpretation of data. Rania Khalil provided intellectual content of critical importance to the work and technical support. Moustafa Abdelsalam participated in the study design and coordination and helped to draft the manuscript. All authors read and approved the final manuscript.

\section{References}

[1] Hutter MM, Schirmer BD, Jones DB, et al. First report from the American College of Surgeons Bariatric Surgery Center Network: laparoscopic sleeve gastrectomy has morbidity and effectiveness positioned between the band and the bypass. Ann Surg 2011;254:410-420.

[2] Lee SY, Lim CH, Pasupathy S, et al. Laparoscopic sleeve gastrectomy: a novel procedure for weight loss. Singapore Med J 2011;52:794-800.

[3] Yousseif A, Emmanuel J, Karra E, et al. Differential effects of 
laparoscopic sleeve gastrectomy and laparoscopic gastric bypass on appetite, circulating acyl-ghrelin, peptide YY336 and active GLP-1 levels in non-diabetic humans. Obes Surg 2014;24:241-252.

[4] Aggarwal S, Kini SU, Herron DM. Laparoscopic sleeve gastrectomy for morbid obesity: a review. Surg Obes Relat Dis 2007;3:189-194.

[5] Bohdjalian A, Langer FB, Shakeri-Leidenmühler S, et al. Sleeve gastrectomy as sole and definitive bariatric procedure: 5-year results for weight loss and ghrelin. Obes Surg 2010;20:535-540.

[6] Himpens J, Dobbeleir J, Peeters G. Long-term results of laparoscopic sleeve gastrectomy for obesity. Ann Surg 2010; 252:319-324.

[7] Kaska L, Sledzinski T, Chomiczewska A, Dettlaff-Pokora A, Swierczynski J. Improved glucose metabolism following bariatric surgery is associated with increased circulating bile acid concentrations and remodeling of the gut microbiome. World J Gastroenterol 2016;22:8698-8719.

[8] Nauta FL, Boertien WE, Bakker SJ, et al. Glomerular and tubular damage markers are elevated in patients with diabetes. Diabetes Care 2011;34:975-981.

[9] Nejat M, Pickering JW, Walker RJ, Endre ZH. Rapid detection of acute kidney injury by plasma cystatin $\mathrm{C}$ in the intensive care unit. Nephrol Dial Transplant 2010;25:32833289.

[10] de Geus HR, Bakker J, Lesaffre EM, le Noble JL. Neutrophil gelatinase-associated lipocalin at ICU admission predicts for acute kidney injury in adult patients. Am J Respir Crit Care Med 2011;183:907-914.

[11] Liang XL, Liu SX, Chen YH, et al. Combination of urinary kidney injury molecule-1 and interleukin-18 as early biomarker for the diagnosis and progressive assessment of acute kidney injury following cardiopulmonary bypass surgery: a prospective nested case-control study. Biomarkers 2010;15:332-339.

[12] Emlet DR, Pastor-Soler N, Marciszyn A, et al. Insulin-like growth factor binding protein 7 and tissue inhibitor of metalloproteinases-2: differential expression and secretion in human kidney tubule cells. Am J Physiol Renal Physiol 2017;312:F284-F296.

[13] Vaidya VS, Ramirez V, Ichimura T, Bobadilla NA, Bonventre JV. Urinary kidney injury molecule-1: a sensitive quantitative biomarker for early detection of kidney tubular injury. Am J Physiol Renal Physiol 2006;290:F517-F529.

[14] Yin C, Wang N. Kidney injury molecule-1 in kidney disease.
Ren Fail 2016;38:1567-1573.

[15] Hanas R, John G; International HbA(1c) Consensus Committee. 2010 Consensus statement on the worldwide standardization of the hemoglobin A(1c) measurement. Clin Chem Lab Med 2010;48:775-776.

[16] Talke H, Schubert GE. [Enzymatic urea determination in the blood and serum in the warburg optical test]. Klin Wochenschr 1965;43:174-175. German.

[17] Kageyama N. A direct colorimetric determination of uric acid in serum and urine with uricase-catalase system. Clin Chim Acta 1971;31:421-426.

[18] Bartels H, Böhmer M, Heierli C. [Serum creatinine determination without protein precipitation]. Clin Chim Acta 1972;37:193-197. German.

[19] Levey AS, Bosch JP, Lewis JB, Greene T, Rogers N, Roth D. A more accurate method to estimate glomerular filtration rate from serum creatinine: a new prediction equation. Modification of diet in renal disease study group. Ann Intern Med 1999;130:461-470.

[20] Salazar DE, Corcoran GB. Predicting creatinine clearance and renal drug clearance in obese patients from estimated fat-free body mass. Am J Med 1988;84:1053-1060.

[21] Landau S, Everitt BS. A handbook of statistical analyses using SPSS. Boca Raton: Chapman \& Hall/CRC; 2004.

[22] English WJ, DeMaria EJ, Brethauer SA, Mattar SG, Rosenthal RJ, Morton JM. American Society for Metabolic and Bariatric Surgery estimation of metabolic and bariatric procedures performed in the United States in 2016. Surg Obes Relat Dis 2018;14:259-263.

[23] Mingrone G, Panunzi S, De Gaetano A, et al. Bariatric surgery versus conventional medical therapy for type 2 diabetes. N Engl J Med 2012;366:1577-1585.

[24] Schauer PR, Bhatt DL, Kirwan JP, et al.; STAMPEDE Investigators. Bariatric surgery versus intensive medical therapy for diabetes - 5-year outcomes. N Engl J Med 2017;376:641651.

[25] Sachdev P, Makaya T, Marven SS, Ackroyd R, Wales JK, Wright NP. Bariatric surgery in severely obese adolescents: a single-centre experience. Arch Dis Child 2014;99:894-898.

[26] Kalinowski P, Paluszkiewicz R, Wróblewski T, et al. Ghrelin, leptin, and glycemic control after sleeve gastrectomy versus Roux-en-Y gastric bypass-results of a randomized clinical trial. Surg Obes Relat Dis 2017;13:181-188.

[27] Adams ST, Salhab M, Hussain ZI, Miller GV, Leveson SH. Roux-en-Y gastric bypass for morbid obesity: what are the preoperative predictors of weight loss? Postgrad Med J 
2013;89:411-416.

[28] Jambhekar A, Maselli A, Robinson S, Kabata K, Gorecki P. Demographics and socioeconomic status as predictors of weight loss after laparoscopic sleeve gastrectomy: a prospective cohort study. Int J Surg 2018;54(Pt A):163-169.

[29] Benaiges D, Flores Le-Roux JA, Pedro-Botet J, et al. Sleeve gastrectomy and Roux-en-Y gastric bypass are equally effective in correcting insulin resistance. Int J Surg 2013;11:309313.

[30] Holcomb CN, Goss LE, Almehmi A, Grams JM, Corey BL. Bariatric surgery is associated with renal function improvement. Surg Endosc 2018;32:276-281.

[31] Navarro-Díaz M, Serra A, Romero R, et al. Effect of drastic weight loss after bariatric surgery on renal parameters in extremely obese patients: long-term follow-up. J Am Soc Nephrol 2006;17(12 Suppl 3):S213-S217.

[32] Kim EY, Kim YJ. Does bariatric surgery really prevent deterioration of renal function? Surg Obes Relat Dis 2016;12:856861.

[33] Neff KJ, Frankel AH, Tam FW, Sadlier DM, Godson C, le Roux CW. The effect of bariatric surgery on renal function and disease: a focus on outcomes and inflammation. Nephrol Dial Transplant 2013;28 Suppl 4:iv73-iv82.

[34] Park S, Kim YJ, Choi CY, Cho NJ, Gil HW, Lee EY. Bariatric surgery can reduce albuminuria in patients with severe obesity and normal kidney function by reducing systemic inflammation. Obes Surg 2018;28:831-837.

[35] Scheven L, Joosten MM, de Jong PE, Bakker SJ, Gansevoort RT; PREVEND study group. The association of albuminuria with tubular reabsorption of uric acid: results from a general population cohort. J Am Heart Assoc 2014;3:e000613.

[36] Goknar N, Oktem F, Ozgen IT, et al. Determination of early urinary renal injury markers in obese children. Pediatr Nephrol 2015;30:139-144.

[37] Thomas MC, Burns WC, Cooper ME. Tubular changes in early diabetic nephropathy. Adv Chronic Kidney Dis 2005; 12:177-186.

[38] Xiao N, Devarajan P, Inge TH, Jenkins TM, Bennett M, Mitsnefes MM. Subclinical kidney injury before and 1 year after bariatric surgery among adolescents with severe obesity. Obesity (Silver Spring) 2015;23:1234-1238.

[39] Chang AR, Grams ME, Navaneethan SD. Bariatric surgery and kidney-related outcomes. Kidney Int Rep 2017;2:261270

[40] Li K, Zou J, Ye Z, et al. Effects of bariatric surgery on renal function in obese patients: a systematic review and meta analysis. PLoS One 2016;11:e0163907. 